BOOK BRIEFLY NOTED

\section{Operative Neuromodulation Vol. 2: Neural Networks Surgery (Acta Neurochirurgica Supplementum), Supplement 97/2}

D.E. Sakas and B.A. Simpson, eds. New York: SpringerWien; 2007, 590 pages, 179 illustrations, \$259.00.

$\mathbf{T}$ his 590-page book on operative neuromodulation comprises a series of papers covering the use of stimulation (predominantly electrical) in major neurologic and psychiatric diseases. Covered is an introductory chapter, which ex- plains, among other things, what is meant by neuromodulation and neural networks, what exactly can be or is stimulated, and how nanotechnology will advance the field. Details of neuromodulation in the treatment/evaluation of pain syndromes, epilepsy, visual and hearing disorders, psychiatric diseases, and movement disorders let the reader know where much of neurosurgery is heading. The neuroradiologist could gain an appreciation of the loci of stimulation, presume what complications may be encountered, and, most important, see how neurosurgery is delving into areas that, heretofore, have been out of their primary purview. Furthermore, because prestimulation imaging (CT/MR) is performed, knowledge of the target in each disease will make the neuroradiologist increasingly valuable in this growing area of patient treatment.

DOl 10.3174/ajnr.A0942 